\title{
Componentwise ultimate bound computation for switched linear systems
}

\author{
Hernan Haimovich and María M. Seron
}

\begin{abstract}
We present a novel ultimate bound computation method for switched linear systems with disturbances and arbitrary switching. We consider both discrete-time and continuoustime systems. The proposed method relies on the existence of a transformation that takes all matrices of the switched linear system into a convenient form satisfying certain properties. The method provides ultimate bounds in the form of polyhedral sets and/or mixed ellipsoidal/polyhedral sets, and it is completely systematic once the aforementioned transformation is obtained. We show that the transformation can be found in the wellknown case where the matrices of the switched linear system generate a solvable Lie algebra. In the latter case, our results also constitute a new sufficient condition for practical stability. An example comparing the bounds obtained by the proposed ultimate bound computation method with those obtained from a common quadratic Lyapunov function computed via linear matrix inequalities shows a clear advantage of the proposed method in some cases.
\end{abstract}

\section{INTRODUCTION}

Switched systems are a special type of dynamical systems that combine a finite number of subsystems by means of a switching rule [17], [14]. Switched systems constitute a convenient description for many systems of practical importance, including many industrial processes, aircraft control, control of mechanical systems in general, and power systems. If all subsystems of a switched system are continuous-time (discrete-time) dynamical systems, we say that the switched system is a switched continuous-time (discrete-time) system. In addition, if all subsystems are linear, we refer to the switched system as a switched linear system.

The stability and stabilisability of switched systems is an area where considerable research effort has been spent in recent years [15], [3], [22], [17]. Different stability problems for switched systems arise depending on whether stability should hold for every admissible switching signal (arbitrary switching), for every switching signal within some class (constrained switching) or for a specific switching signal (switching stabilisation). This paper focuses on the arbitrary switching case.

In general, most attention was devoted to analysing or ensuring the asymptotic stability of an equilibrium point for the switched system [15], [3], [22], [17]. However, there exist numerous reasons why asymptotic stability may be prevented in a realistic setting. One such reason is that switching may be employed to drive the state of the switched

H. Haimovich is with CONICET and Laboratorio de Sistemas Dinámicos y Procesamiento de Información, Departamento de Control, Facultad de Cs. Exactas, Ingeniería y Agrimensura, Universidad Nacional de Rosario, Argentina. h.haimovich@gmail.com

M.M. Seron is with the Centre for Complex Dynamic Systems \& Control, The University of Newcastle, Australia. maria.seron@newcastle.edu.au system close to a point that is not an equilibrium point of all subsystems [25]. Another reason is that nonvanishing perturbations (also named persistent disturbances) may act on the system [10, Ch. 9]. When asymptotic stability is not possible, ensuring some type of practical stability such as the ultimate boundedness of the state trajectories becomes important.

Some results have been reported on the practical stability of switched systems. In [16], a networked control system with delay and packet dropout is modelled as a switched discrete-time system where the properties of interest (stability and $l^{\infty}$ persistent disturbance attenuation) have to be analysed for the arbitrary switching case. In [23], a switched discrete-time system is considered where the switching is state-dependent and the problem is that of finding controls that steer the state from a set of initial states to a set of "safe" states. References [28] and [27] address control design to ensure uniform ultimate boundedness for switched linear systems with parametric uncertainties under arbitrary switching by means of a common Lyapunov function approach. In [29], the authors address the design of both the control and switching strategy to achieve uniform ultimate boundedness of the system state.

Most existing ultimate bound computation methods either make use of level sets of a Lyapunov function or employ some norm of the system state to compute the ultimate bound set. For switched linear systems, a quadratic Lyapunov function common to all subsystems can be computed via linear matrix inequalities (LMIs) in case it exists (see, for example, Section 4.3 of [22] and the references therein).

In this paper, we address the computation of ultimate bounds for both switched discrete-time and switched continuous-time linear systems. We derive a novel ultimate bound computation method that is based on componentwise analysis and extends to switched systems previous results presented by the authors in [12] and [8]. The method requires neither the computation of a Lyapunov function for the system nor the use of a norm for the system state. We provide an example where our method is able to yield ultimate bounds that are tighter than those obtained by means of a common quadratic Lyapunov function computed via LMIs. A feature of the method is that the ultimate bounds obtained are of a mixed polyhedral/ellipsoidal form. In addition, our method becomes completely systematic in some cases.

The componentwise ultimate-bound computation method of [12], [8] has been successfully applied to the analysis of sampled-data systems involving quantisation [7], to the computation of the error introduced by PWM signals in LTI systems [11], and to the development of new controller 
design methods [13]. Moreover, a novel application in fault tolerant control systems has been recently reported in [21], [18], [20] and [26]. In these papers, the ultimate-bound computation method of [12] has been employed to obtain invariant sets where the system behaviour under "healthy" and "faulty" operation can be confined; thus fault tolerance can be achieved whenever those sets are "separated" in some sense. In particular, [21] proposed a multisensor switching strategy that takes advantage of the aforementioned set separation to automatically avoid the use of faulty sensors in the closed-loop system. Thus, the results presented in the current paper have relevance in fault tolerant control systems and we envisage their application in the analysis and design of improved multisensor switching strategies with fault tolerance guarantees.

\section{Notation}

$\mathbb{R}$ and $\mathbb{C}$ denote the sets of real and complex numbers, respectively. If $x(t)$ is a vector-valued function, then $\lim \sup _{t \rightarrow \infty} x(t)$ denotes the vector obtained by taking limsup $\operatorname{su}_{t \rightarrow \infty}$ of each component of $x(t)$. Similarly, 'max' denotes componentwise maximum. $|M|$ and $\mathbb{R e}(M)$ denote the elementwise magnitude and real part, respectively, of a matrix or vector $M$. The $(i, k)$-th entry of a matrix $M$ is denoted by $M_{i, k}$. If $X, Y \in \mathbb{R}^{n \times m}$, the expression ' $X \preceq Y$ ' denotes the set of componentwise inequalities $X_{i, k} \leq Y_{i, k}$, $i=1, \ldots, n, k=1, \ldots, m$, between the entries of $X$ and $Y$, and similarly for $X \succeq Y . \mathbb{R}_{+}$denotes the nonnegative real numbers, $\mathbb{N}_{0}$ the nonnegative integers, and $j$ the imaginary unit $\left(j^{2}=-1\right)$. Given a matrix $M \in \mathbb{R}^{n \times n}, \boldsymbol{\rho}(M)$ denotes its spectral radius, that is, the maximum magnitude of its eigenvalues. A matrix $M \in \mathbb{R}^{n \times n}$ is Metzler if $M_{i, k} \geq 0$ for all $i \neq k . M$ is Metzler if and only if $e^{M t} \succeq 0$ for all $t \geq 0$. Given an arbitrary matrix $N \in \mathbb{C}^{n \times n}$, we define $\mathcal{M}(N) \in \mathbb{R}^{n \times n}$ as the matrix whose entries satisfy

$$
[\mathcal{M}(N)]_{i, k}= \begin{cases}\mathbb{R e}\left\{N_{i, k}\right\} & \text { if } i=k, \\ \left|N_{i, k}\right| & \text { if } i \neq k .\end{cases}
$$

Note that $\mathcal{M}(N)$ is Metzler for every $N \in \mathbb{C}^{n \times n}$.

\section{Switched Discrete-TIMe Systems}

We consider the linear discrete-time switched system

$$
x_{k+1}=A_{\sigma(k)} x_{k}+E_{\sigma(k)} w_{k},
$$

where $x_{k} \in \mathbb{R}^{n}$ is the system state, $w_{k} \in \mathbb{R}^{p}$ is a perturbation, and

$$
\sigma: \mathbb{N}_{0} \rightarrow\{1,2, \ldots, \mathrm{s}\}
$$

is the switching function. The evolution of the perturbation $w_{k}$ is unknown but assumed to have a componentwise bound

$$
\left|w_{k}\right| \preceq \mathbf{w}, \quad \text { for all } k \geq 0,
$$

where $\mathbf{w} \in \mathbb{R}_{+}^{p}$ is a known constant vector. In the following theorem we derive an ultimate bound on the switched system state that is valid for any realization of the switching function $\sigma$. In addition, the derived ultimate bound takes the componentwise form of the perturbation bound (4) into account.
Theorem 1: Consider the linear switched discrete-time system (2) with switching function (3) and componentwise perturbation bound (4). Suppose that an invertible transformation $V \in \mathbb{C}^{n \times n}$ exists such that

$$
\Lambda \triangleq \max _{i=1,2, \ldots, \mathrm{S}}\left|V^{-1} A_{i} V\right|
$$

satisfies $\rho(\Lambda)<1$. Define

$$
\mathbf{z} \triangleq \max _{i=1,2, \ldots, \mathrm{S}}\left[\max _{w:|w| \succeq \mathbf{w}}\left|V^{-1} E_{i} w\right|\right] .
$$

Then,

$$
\begin{aligned}
& \limsup _{k \rightarrow \infty}\left|V^{-1} x_{k}\right| \preceq(\mathrm{I}-\Lambda)^{-1} \mathbf{z}, \text { and } \\
& \limsup _{k \rightarrow \infty}\left|x_{k}\right| \preceq|V|(\mathrm{I}-\Lambda)^{-1} \mathbf{z} .
\end{aligned}
$$

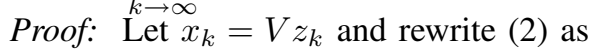

$$
z_{k+1}=V^{-1} A_{\sigma(k)} V z_{k}+V^{-1} E_{\sigma(k)} w_{k} .
$$

Taking componentwise magnitudes in (9) and operating yields

$$
\left|z_{k+1}\right| \preceq\left|V^{-1} A_{\sigma(k)} V\right|\left|z_{k}\right|+\left|V^{-1} E_{\sigma(k)} w_{k}\right| .
$$

Note that, for all $k,\left|V^{-1} A_{\sigma(k)} V\right| \preceq \Lambda$, and

$$
\left|V^{-1} E_{\sigma(k)} w_{k}\right| \preceq \max _{w:|w| \preceq \mathbf{w}}\left|V^{-1} E_{\sigma(k)} w\right| \preceq \mathbf{z} .
$$

Define the auxiliary system

$$
y_{k+1}=\Lambda y_{k}+\mathbf{z}, \quad y_{0} \triangleq\left|z_{0}\right|=\left|V^{-1} x_{0}\right| .
$$

From (9)-(12) it follows that $\left|z_{k}\right| \preceq y_{k}$ for all $k$. Therefore,

$$
\limsup _{k \rightarrow \infty}\left|z_{k}\right| \preceq \limsup _{k \rightarrow \infty} y_{k}=\lim _{k \rightarrow \infty} y_{k}=(\mathrm{I}-\Lambda)^{-1} \mathbf{z},
$$

where the last equality above follows from (12) and since $\boldsymbol{\rho}(\Lambda)<1$. Using (13) and recalling that $z_{k}=V^{-1} x_{k}$, then (7) follows, and (8) is established from (7) and the fact that $|x| \preceq|V|\left|V^{-1} x\right|$.

Remark 1: The computation of the maximum between square brackets in (6) requires the solution to the $n$ optimization problems $\max _{|w| \preceq \mathbf{w}}\left|V^{-1} E_{i} w\right|$. The solution to these problems can be easily obtained as follows. Express the matrix $V^{-1} E_{i} \in \mathbb{C}^{n \times p}$ as $W \triangleq V^{-1} E_{i}=X+j Y$, where $X=\mathbb{R e}\left(V^{-1} E_{i}\right)$ and $Y=\mathbb{I m}\left(V^{-1} E_{i}\right)$. Let $W_{j}^{T}, X_{j}^{T}$, and $Y_{j}^{T}$ denote the $j$-th rows of $W, X$, and $Y$, respectively, and note then that $\left|V^{-1} E_{i} w\right|=\left[\left|W_{1}^{T} w\right| \ldots\left|W_{n}^{T} w\right|\right]^{T}$. We have

$$
\left|W_{j}^{T} w\right|=\sqrt{w^{T}\left(X_{j} X_{j}^{T}+Y_{j} Y_{j}^{T}\right) w} .
$$

Therefore, $\max _{|w| \preceq \mathbf{w}}\left|V^{-1} E_{i} w\right|$ is a set of $n$ constrained convex maximization problems, each over the convex and bounded polyhedral constraint set $C \triangleq\left\{w \in \mathbb{R}^{p}:|w| \preceq\right.$ $\mathbf{w}\}$. Therefore, its solution can be found by evaluating each component of $\left|V^{-1} E_{i} w\right|$ on the extreme points of $C$, and selecting the maximum value per component (see, for example, [2]).

Remark 2: The ultimate bounds of the form (7) have polyhedral shape if the transformation matrix $V$ is real and 
(possibly degenerate) ellipsoidal shape if $V$ has complex entries (see [8] for details).

Theorem 1 derives an ultimate bound of the form (7) for the switched system (2)-(4). The procedure is completely systematic [see (5), (6) and Remark 1] once a transformation $V$ is found such that the (elementwise) maximum among the magnitudes of the transformed matrices $V^{-1} A_{i} V$, for $i=1, \ldots, \mathrm{s}$, has spectral radius smaller than one. The following subsection shows that such a transformation can be found in some special cases of interest.

\section{A. Special Cases}

1) Single system $(\mathrm{S}=1)$ : An LTI system of the form

$$
x_{k+1}=A x_{k}+E w_{k}
$$

can be regarded as a special case of (2) where the switching function $\sigma$ is constant. In this case, let $V \in \mathbb{C}^{n \times n}$ be the transformation that takes $A$ into its Jordan canonical form $\Lambda_{1}=V^{-1} A V$ and note that $\Lambda=\left|\Lambda_{1}\right|$ [see (5)] has spectral radius less than one if and only $A$ has spectral radius less than one (since the diagonal entries of a matrix in Jordan form are its eigenvalues). Therefore, the hypotheses of Theorem 1 are satisfied if and only if $A$ has spectral radius less than one, which is a well-known condition for system (14) to have an ultimate bound. The ultimate bound expression (7) becomes in this case

$$
\limsup _{k \rightarrow \infty}\left|V^{-1} x_{k}\right| \preceq\left(\mathrm{I}-\left|\Lambda_{1}\right|\right)^{-1}\left[\max _{w:|w| \preceq \mathbf{w}}\left|V^{-1} E w\right|\right],
$$

which is consistent with the results of [12] and [8].

2) $A_{1}, A_{2}, \ldots, A_{\mathrm{s}}$ generate a solvable Lie algebra: The fact that the perturbed switching system (2) has an ultimate bound implies that the nominal switching system

$$
x_{k+1}=A_{\sigma(k)} x_{k}
$$

is asymptotically stable or, at least, should also have an ultimate bound. Note also that the stability of (15) for arbitrary switching implies the stability of each of the derived non-switching subsystems $x_{k+1}=A_{i} x_{k}$, for $i=1,2, \ldots, \mathrm{s}$. Well-known results in the area of switched systems relate the stability properties of (15) with the Lie algebra generated by the matrices $A_{1}, A_{2}, \ldots, A_{\mathrm{S}}$ [5], [24]. In particular, it is known that if (i) each of these matrices has spectral radius less than one and (ii) the Lie algebra generated by these matrices is solvable, then (15) is stable for arbitrary switching. The following result shows that if the above conditions (i) and (ii) hold, then a transformation exists that satisfies the hypothesis of Theorem 1.

Lemma 1: Consider the linear switched discrete-time system (2) with switching function (3). Suppose that $\boldsymbol{\rho}\left(A_{i}\right)<1$ for $i=1,2, \ldots, \mathrm{S}$ and that the Lie algebra generated by $A_{1}$, $A_{2}, \ldots, A_{\mathrm{S}}$ is solvable. Then, an invertible $V \in \mathbb{C}^{n \times n}$ exists such that $\rho(\Lambda)<1$, with $\Lambda$ as defined in (5).

Proof: It is a consequence of Lie's theorem (see for example [4]) that if the Lie algebra generated by $A_{1}, A_{2}$, $\ldots, A_{\mathrm{S}}$ is solvable then there exists $V \in \mathbb{C}^{n \times n}$ such that $V^{-1} A_{i} V$ is upper triangular for $i=1,2, \ldots, \mathrm{s}$. Then,
$\left|V^{-1} A_{i} V\right|$ also is upper triangular. Since the eigenvalues of a triangular matrix are the elements of the main diagonal, then $\boldsymbol{\rho}\left(A_{i}\right)=\boldsymbol{\rho}\left(V^{-1} A_{i} V\right)=\boldsymbol{\rho}\left(\left|V^{-1} A_{i} V\right|\right)<1$ for $i=1,2, \ldots$, S. Therefore $\rho(\Lambda)<1$ and $V$ is the required transformation.

Remark 3: Lemma 1 ensures that Theorem 1 is applicable in the well-reported case that the subsystem matrices $A_{i}$ generate a solvable Lie algebra. In fact, it extends existing stability results, which apply to the nominal system (15), to the case of systems having bounded perturbations as in (2)-(4). As is well-known [14], the solvable Lie algebra condition for stability is not robust, since arbitrarily small perturbations of the entries of the subsystem matrices may destroy the solvability property. However, note that the result of Theorem 1 is indeed robust in this sense.

\section{SWitched Continuous-Time Systems}

Consider the continuous-time switched system

$$
\dot{x}(t)=A_{\sigma(t)} x(t)+E_{\sigma(t)} w(t),
$$

where $x(t) \in \mathbb{R}^{n}$ is the system state, $w(t) \in \mathbb{R}^{p}$ is a perturbation, and

$$
\sigma: \mathbb{R}_{+} \rightarrow\{1,2, \ldots, s\}
$$

is the piecewise constant switching function, assumed to have a finite number of discontinuities in every bounded interval. The evolution of the perturbation $w$ is unknown but assumed to have a componentwise bound

$$
|w(t)| \preceq \mathbf{w}, \quad \text { for all } t \geq 0,
$$

where $\mathbf{w} \in \mathbb{R}_{+}^{p}$ is a known constant vector.

Similarly to the discrete-time case, we will derive ultimate bounds on the switched continuous-time system state that are valid for any realization of the switching function $\sigma$ and, in addition, can take the componentwise form of the perturbation bound (18) into account.

The main result of the section is derived in Theorem 2, which is preceded by two auxiliary Lemmas. The latter are comparison results that show that the solution of a linear differential equation of certain characteristics (namely, whose evolution matrix is either bounded by a Metzler matrix or is itself a Metzler matrix) can be bounded by the solution of a differential equation of simpler form.

Lemma 2: Consider a real continuous-time system of the form

$$
\dot{x}(t)=A(t) x(t)+v(t),
$$

where the time-varying matrix $A(t) \in \mathbb{R}^{n \times n}$ and the independent term $v(t) \in \mathbb{R}^{n}$ are such that $x(t) \succeq 0$ for all $t \geq 0$ whenever $x(0) \succeq 0$. Let $B \in \mathbb{R}^{n \times n}$ be Metzler and satisfy $B-A(t) \succeq 0$ for all $t \geq 0$, and consider the auxiliary system

$$
\dot{y}(t)=B y(t)+v(t) .
$$

Then, $y(t) \succeq x(t)$ for all $t \geq 0$ provided $y(0) \succeq x(0) \succeq 0$.

Proof: Define $z \triangleq y-x$. From (19) and (20) it follows that

$$
\dot{z}(t)=B z(t)+(B-A(t)) x(t) .
$$


The solution to (21) can be written as

$$
z(t)=e^{B t} z(0)+\int_{0}^{t} e^{B(t-\tau)}(B-A(\tau)) x(\tau) d \tau .
$$

Since $B$ is Metzler, then $e^{B t} \succeq 0$ for all $t \geq 0$. Also, $z(0)=$ $y(0)-x(0) \succeq 0, B-A(t) \succeq 0$ for all $t \geq 0$, and $x(t) \succeq 0$ for all $t \geq 0$, all by assumption. Using these facts in (22) shows that $z(t) \succeq 0$ whence $y(t) \succeq x(t)$, for all $t \geq 0$.

Lemma 3: Consider a real continuous-time system

$$
\dot{x}(t)=A x(t)+v(t),
$$

where $A \in \mathbb{R}^{n \times n}$ is Metzler and the independent term $v(t) \in$ $\mathbb{R}^{n}$ satisfies $|v(t)| \preceq \mathbf{w}$, for all $t \geq 0$. Define the auxiliary system $\dot{y}(t)=A y(t)+\mathbf{w}$. Then, $y(t) \succeq x(t)$ for all $t \geq 0$ provided $y(0) \succeq x(0)$.

Proof: Define $z \triangleq y-x$. Then $\dot{z}(t)=A z(t)+p(t)$, where $p(t) \triangleq \mathbf{w}-v(t)$ satisfies $p(t) \succeq 0$ since $|v(t)| \preceq \mathbf{w}$. We have

$$
z(t)=e^{A t} z(0)+\int_{0}^{t} e^{A(t-\tau)} p(\tau) d \tau .
$$

Since $A$ is Metzler, then $e^{A t} \succeq 0$ for all $t \geq 0$. Also, $z(0) \succeq$ 0 and $p(t) \succeq 0$ for all $t \geq 0$. Using these facts in (24) yields $z(t) \succeq 0$, whence $y(t) \succeq x(t)$, for all $t \geq 0$.

Theorem 2: Consider the switched system (16) with switching function (17) and componentwise perturbation bound (18). Suppose that an invertible transformation $V \in$ $\mathbb{C}^{n \times n}$ and a Metzler and Hurwitz ${ }^{1}$ matrix $\Lambda \in \mathbb{R}^{n \times n}$ exist such that $\Lambda-\mathcal{M}\left(\Lambda_{i}\right) \succeq 0$, with

$$
\Lambda_{i} \triangleq V^{-1} A_{i} V,
$$

for $i=1, \ldots, \mathrm{s}$, and where $\mathcal{M}(\cdot)$ is the operation defined in (1), and recall $\mathbf{z}$ as defined in (6). Then,

$$
\limsup _{t \rightarrow \infty}\left|V^{-1} x(t)\right| \preceq-\Lambda^{-1} \mathbf{z} .
$$

Proof: Let $\stackrel{t \rightarrow \infty}{x=V z}$ and rewrite (16) as

$$
\dot{z}(t)=\Lambda_{\sigma(t)} z(t)+V^{-1} E_{\sigma(t)} w(t) .
$$

Since $z$ may have complex components, write $z(t)=$ $e^{j \Theta(t)} \rho(t)$ with $\rho(t)=|z(t)|$ and

$$
\Theta(t)=\operatorname{diag}\left(\theta_{1}(t), \ldots, \theta_{n}(t)\right), \quad \theta_{k}(t)=\arg \left\{z_{k}(t)\right\} .
$$

Substituting in (27) yields

$$
\begin{aligned}
\dot{z}(t) & =j e^{j \Theta(t)} \dot{\Theta}(t) \rho(t)+e^{j \Theta(t)} \dot{\rho} \\
& =\Lambda_{\sigma(t)} e^{j \Theta(t)} \rho(t)+V^{-1} E_{\sigma(t)} w(t) .
\end{aligned}
$$

Multiplication on the left by $e^{-j \Theta(t)}$ yields

$$
\begin{aligned}
& j \dot{\Theta}(t) \rho(t)+\dot{\rho}(t)= \\
& \quad e^{-j \Theta(t)} \Lambda_{\sigma(t)} e^{j \Theta(t)} \rho(t)+e^{-j \Theta(t)} V^{-1} E_{\sigma(t)} w(t),
\end{aligned}
$$

and taking real parts, it follows that

$$
\begin{aligned}
\dot{\rho}(t)= & \mathbb{R e}\left\{e^{-j \Theta(t)} \Lambda_{\sigma(t)} e^{j \Theta(t)}\right\} \rho(t)+ \\
& \mathbb{R e}\left\{e^{-j \Theta(t)} V^{-1} E_{\sigma(t)} w(t)\right\} .
\end{aligned}
$$

\footnotetext{
${ }^{1}$ A matrix $\Lambda \in \mathbb{R}^{n \times n}$ is Metzler and Hurwitz if and only if $-\Lambda$ is an M-matrix. See [9] for the definition and properties of M-matrices.
}

Define

$$
F(t) \triangleq \mathbb{R e}\left\{e^{-j \Theta(t)} \Lambda_{\sigma(t)} e^{j \Theta(t)}\right\} .
$$

From (32) and (28), we have

$$
[F(t)]_{i, k}= \begin{cases}\mathbb{R e}\left\{\left[\Lambda_{\sigma(t)}\right]_{i, k}\right\} & \text { if } i=k, \\ \mathbb{R e}\left\{e^{-j\left(\theta_{i}(t)-\theta_{k}(t)\right)}\left[\Lambda_{\sigma(t)}\right]_{i, k}\right\} & \text { if } i \neq k,\end{cases}
$$

whence $F(t) \preceq \mathcal{M}\left(\Lambda_{\sigma(t)}\right)$. Since $\mathcal{M}\left(\Lambda_{\sigma(t)}\right) \preceq \Lambda$, it follows that $F(t) \preceq \Lambda$. Since $\rho(t)=|z(t)| \geq 0$ by definition, Lemma 2 then asserts that $\rho(t) \preceq y(t)$, where $y(t)$ satisfies $\dot{y}(t)=\Lambda y(t)+\gamma(t)$, with

$$
\gamma(t) \triangleq \mathbb{R e}\left\{e^{-j \Theta(t)} V^{-1} E_{\sigma(t)} w(t)\right\} .
$$

We have $|\gamma(t)| \preceq\left|V^{-1} E_{\sigma(t)} w(t)\right| \preceq \mathbf{z}$. From Lemma 3, then $\rho(t) \preceq y(t)$ with $\dot{y}(t)=\Lambda y(t)+\mathbf{z}$ and provided $y(0) \succeq \rho(0)$. Then,

$$
\begin{aligned}
\limsup _{t \rightarrow \infty}\left|V^{-1} x(t)\right|=\limsup _{t \rightarrow \infty} \rho(t) \\
\preceq \limsup _{t \rightarrow \infty} y(t)=\lim _{t \rightarrow \infty} y(t)=-\Lambda^{-1} \mathbf{z},
\end{aligned}
$$

where we have used the fact that $\Lambda$ is Hurwitz. The result is thus established.

Theorem 2 presents a systematic method to compute an ultimate bound for the continuous-time switched system (16)-(18). The method relies on the existence of a transformation $V$ such that a function [given by the operation $\mathcal{M}(\cdot)$ defined in (1)] of the transformed matrices $V^{-1} A_{i} V$, for $i=1, \ldots, \mathrm{S}$, is bounded by a Metzler Hurwitz matrix. The following subsection shows that such a transformation can be found in some special cases of interest.

\section{A. Special Cases}

1) Single system $(\mathrm{S}=1)$ : The case of an LTI system of the form

$$
\dot{x}(t)=A x(t)+E w(t)
$$

can be regarded as a special case of (16) where the switching function $\sigma$ is constant. As in Section II-A.1, let $V \in \mathbb{C}^{n \times n}$ be the transformation that takes $A$ into its Jordan canonical form $\Lambda_{1}=V^{-1} A V$ and note that $\Lambda=\mathcal{M}\left(\Lambda_{1}\right)=\mathbb{R e}\left(\Lambda_{1}\right)$, since the off-diagonal entries of a matrix in Jordan form are always real and positive. Also, note that $A$ is Hurwitz if and only if $\Lambda_{1}$ is, which in turn happens if and only if $\Lambda=\operatorname{Re}\left(\Lambda_{1}\right)$ also is Hurwitz. Therefore, the hypotheses of Theorem 2 are satisfied if and only if $A$ is Hurwitz, which is a well-known condition for system (35) to have an ultimate bound. Note that, in this case, the hypotheses of Theorem 2 incur no loss of generality.

Next, noting that the inverse of a Jordan matrix with negative real eigenvalues is a matrix with nonpositive elements, we can write $-\Lambda^{-1}=-\left[\mathbb{R e}\left(\Lambda_{1}\right)\right]^{-1}=\left|\left[\mathbb{R e}\left(\Lambda_{1}\right)\right]^{-1}\right|$. Thus, the ultimate bound expression (26) becomes in this case

$$
\limsup _{t \rightarrow \infty}\left|V^{-1} x(t)\right| \preceq\left|\left[\mathbb{R e}\left(\Lambda_{1}\right)\right]^{-1}\right|\left[\max _{w:|w| \preceq \mathbf{w}}\left|V^{-1} E w\right|\right],
$$

which is consistent with the results of [12] and [8]. 
2) $A_{1}, A_{2}, \ldots, A_{\mathrm{S}}$ generate a solvable Lie algebra: Another case of interest is when the matrices $A_{1}, \ldots, A_{\mathrm{S}}$ of system (16) generate a solvable Lie algebra [1]. In this case, let $V \in \mathbb{C}^{n \times n}$ be the transformation that renders $\Lambda_{i}=V^{-1} A_{i} V$ upper triangular for $i=1, \ldots, \mathrm{s}$ and take

$$
\Lambda=\max _{i=1, \ldots, \mathrm{s}} \mathcal{M}\left(\Lambda_{i}\right),
$$

where the maximum is taken componentwise. Note that by definition, $\Lambda$ is Metzler and $\Lambda-\mathcal{M}\left(\Lambda_{i}\right) \succeq 0$ for $i=1, \ldots$, s. Since the $\Lambda_{i}$ are all upper triangular, then the eigenvalues of $\Lambda_{i}$ are its main-diagonal entries, and those of $\mathcal{M}\left(\Lambda_{i}\right)$ are the real parts of those of $\Lambda_{i}$. Note then that $\Lambda$ is Hurwitz if and only if the $\Lambda_{i}$ are all Hurwitz. Therefore, note that also in this case, the hypotheses of Theorem 2 incur no loss of generality.

\section{EXAMPLE}

As an application of our ultimate bound computation method, we consider the equations for a magnetic levitation system, linearised around an equilibrium point:

$$
\dot{x}(t)=A^{c} x(t)+B^{c} u(t)+w(t),
$$

where the components of the system state $x \in \mathbb{R}^{3}$ represent the ball position, ball velocity, and coil current, $u \in \mathbb{R}$ is the input voltage (see [26] for more details), and $w \in \mathbb{R}^{3}$ represents a non-vanishing perturbation, with

$$
A^{c}=\left[\begin{array}{ccc}
0 & 1 & 0 \\
943.27 & 0 & -16.2 \\
0 & 0 & -26.67
\end{array}\right] \quad B^{c}=\left[\begin{array}{c}
0 \\
0 \\
2.4242
\end{array}\right] C=\left[\begin{array}{l}
1 \\
0 \\
0
\end{array}\right]^{T} .
$$

We consider a setting where the magnetic levitation system (37) is sampled at an arbitrarily varying sampling rate subject to the constraint that the sampling period can only assume one of 2 possible values: $10^{-3}$ or $1.37 \cdot 10^{-3}$. If $t_{k}$ for $k=$ $0,1, \ldots$ denote the sampling instants, then such constraint on the possible sampling periods can be expressed as:

$$
t_{k+1}-t_{k} \in\left\{T_{1} \triangleq 10^{-3}, T_{2} \triangleq 1.37 \cdot 10^{-3}\right\} .
$$

This sampling scheme yields the following switched discretetime system:

$$
x_{k+1}=A_{\sigma(k)}^{o} x_{k}+B_{\sigma(k)} u_{k}+w_{k},
$$

where $x_{k}=x\left(t_{k}\right), u_{k}=u\left(t_{k}\right), \sigma: \mathbb{N}_{0} \rightarrow\{1,2\}$, and $w_{k}$ is a discrete-time perturbation derived from the action of $w(t)$ on (37). We assume that $w_{k}$ has the following componentwise bound

$$
\left|w_{k}\right| \preceq\left[\begin{array}{lll}
10^{-5} & 10^{-3} & 10^{-4}
\end{array}\right]^{T} .
$$

The matrices $A_{\sigma(k)}^{o}$ and $B_{\sigma(k)}$ in (40) are given by

$$
A_{\sigma(k)}^{o}=e^{A^{c} T_{\sigma(k)}}, \quad B_{\sigma(k)}=\int_{0}^{T_{\sigma(k)}} e^{A^{c} t} d t B^{c} .
$$

Since the magnetic levitation system is open-loop unstable, stabilisation is one principal control objective. Towards this goal, a switched state feedback control $u_{k}=-K_{\sigma(k)} x_{k}$ is designed, yielding the closed-loop system equation

$$
x_{k+1}=A_{\sigma(k)} x_{k}+w_{k},
$$

where $A_{i} \triangleq A_{i}^{o}-B_{i} K_{i}$ for $i=1,2$. The state-feedback matrices $K_{i}$ for $i=1,2$ are designed so that the closed-loop subsystem matrices $A_{i}$ for $i=1,2$ are stable and generate a solvable Lie algebra. State feedback gains with such a property do not always exist. However, since in this specific example the matrix $A^{c}$ has only one unstable eigenvalue and the matrices $A_{i}^{o}$ are the discrete-time counterparts of $A^{c}$ for different sampling periods, then such feedback matrices are guaranteed to exist (see the companion paper [6] for details). The feedback matrices thus designed are $K_{1}=$ $\left[\begin{array}{lll}-45553 & -1483 & 419\end{array}\right], K_{2}=\left[\begin{array}{lll}-33438 & -1089 & 307\end{array}\right]$, and the simultaneous triangularisation matrix (see Lemma 1 in Section II-A.2) is:

$$
V=\left[\begin{array}{ccc}
-3.254 & 3.302 & 3.254 \\
99.95 & -88.04 & 99.95 \\
0 & 47.30 & 0
\end{array}\right] \cdot 10^{-2} .
$$

Application of Theorem 1 yields

$$
\begin{aligned}
\limsup _{k \rightarrow \infty}\left|V^{-1} x_{k}\right| \preceq\left[\begin{array}{c}
318.1 \\
372.9 \\
0.6398
\end{array}\right] \cdot 10^{-3}, \quad \text { and } \\
\limsup _{k \rightarrow \infty}\left|x_{k}\right| \preceq\left[\begin{array}{c}
22.68 \\
646.8 \\
176.4
\end{array}\right] \cdot 10^{-3} .
\end{aligned}
$$

We next compare the componentwise bound (46) with that obtained via a Lyapunov function. We follow a bounding procedure similar to that in $[10, \mathrm{Ch}$. 9] but adapted to switched discrete-time systems. First, since the feedback matrices $K_{1}$ and $K_{2}$ were designed so that the closed-loop subsystem matrices are stable and generate a solvable Lie algebra, then the existence of a common quadratic Lyapunov function (CQLF) is ensured [14]. The computation of such CQLF for this system may be performed via LMIs. We thus seek $P=P^{T}>0$ so that the following set of LMIs is satisfied:

$$
A_{i}^{T} P A_{i}-e^{-2 \beta T_{i}} P<0,
$$

for $i=1,2$, where the factor $e^{-2 \beta T_{i}}$ is added, similarly to [19], as an additional performance constraint. Such addition leaves $\beta$ as a free parameter. After computing the required ultimate bound for several different values of $\beta$ (including $\beta=0$, which corresponds to stability without any additional performance constraint), we selected $\beta=0.16$ since for such value a smaller ultimate bound could be obtained. Solving the LMIs (47) for this value of $\beta$ yields

$$
P=\left[\begin{array}{ccc}
30209 & 372.4 & -3.693 \\
372.4 & 28.30 & -0.353 \\
-3.693 & -0.353 & 0.060
\end{array}\right]
$$

where the corresponding CQLF is $V(x)=x^{T} P x$. We have

$$
\begin{aligned}
\Delta V_{k} & \triangleq V\left(x_{k+1}\right)-V\left(x_{k}\right) \\
& =x_{k}^{T} Q_{\sigma(k)} x_{k}+2 x_{k}^{T} A_{\sigma(k)}^{T} P w_{k}+w_{k}^{T} P w_{k}
\end{aligned}
$$


where $Q_{i} \triangleq A_{i}^{T} P A_{i}-P$ for $i=1,2$. Operating on (50) yields

$$
\begin{aligned}
& \Delta V_{k} \leq \lambda_{\max }\left(Q_{\sigma(k)}\right)\left\|x_{k}\right\|^{2} \\
&+2\left\|x_{k}\right\|\left\|A_{\sigma(k)}^{T} P w_{k}\right\|+w_{k}^{T} P w_{k} \\
& \leq-\ell_{1}\left\|x_{k}\right\|^{2}+2 \ell_{2}\left\|x_{k}\right\|+\ell_{3}
\end{aligned}
$$

where we have defined

$$
\begin{gathered}
\ell_{1} \triangleq-\max _{i=1,2} \lambda_{\max }\left(Q_{i}\right)=0.04265, \\
\ell_{2} \triangleq \max _{i=1,2}\left[\max _{|w| \preceq \mathbf{w}}\left\|A_{i}^{T} P w\right\|\right]=0.6356, \\
\ell_{3} \triangleq \max _{|w| \preceq \mathbf{w}} w^{T} P w=3.88 \cdot 10^{-5} .
\end{gathered}
$$

The maximum in (55) and that between square brackets in (54) can both easily be obtained by evaluating the corresponding expressions on the vertices of the polyhedral region $|w| \preceq \mathbf{w}$. Since $\ell_{k}>0$ for $k=1,2,3$, then the right-hand side of (52) is not positive if

$$
\left\|x_{k}\right\| \geq \frac{\ell_{2}+\sqrt{\ell_{2}^{2}+\ell_{1} \ell_{3}}}{\ell_{1}}=29.805 .
$$

From (56), we obtain the following ultimate bound set limited by a level curve of the CQLF:

$$
\limsup _{k \rightarrow \infty} x_{k}^{T} P x_{k} \leq \lambda_{\max }(P)(29.805)^{2}=2.684 \cdot 10^{7} \text {. }
$$

It can easily be verified that the ultimate bound (46) is much tighter than that given by (57). It can even be verified that a bound more than 800 times bigger than (46) can still fit inside the region given by (57).

\section{CONCLUSIONS}

We have presented a new ultimate bound computation method for discrete-time and continuous-time switched linear systems with disturbances and arbitrary switching. The proposed method requires a transformation that takes all matrices of the switched linear system into a convenient form satisfying certain properties. The method provides ultimate bounds in the form of polyhedral sets and/or mixed ellipsoidal/polyhedral sets, and it is completely systematic once the aforementioned transformation is obtained. We have shown that the transformation can be found in the wellknown case where the matrices of the switched linear system generate a solvable Lie algebra. We have presented an example which shows that the bounds obtained by the proposed ultimate bound computation method are much tighter than those obtained via a common quadratic Lyapunov function approach. Future work will focus on the application of the results to multisensor switching fault tolerant control.

\section{REFERENCES}

[1] A.A. Agrachev and D. Liberzon. Lie-algebraic stability criteria for switched systems. SIAM J. Control and Optimization, 40(1):253-269, 2001.

[2] J.M. Borwein and A.S. Lewis. Convex Analysis and Nonlinear Optimization. Theory and Examples. Springer, NY, 2000.

[3] R.A. Decarlo, M.S. Branicky, S. Pettersson, and B. Lennartson. Perspectives and results on the stability and stabilizability of hybrid systems. Proc. of the IEEE, 88(7):1069-1082, 2000.
[4] K. Erdmann and M.J. Wildon. Introduction to Lie algebras. SpringerVerlag London, 2006.

[5] L. Gurvits. Stability of discrete linear inclusion. Linear Algebra and its Applications, 231:47-85, 1995.

[6] H. Haimovich, J.H. Braslavsky, and F. Felicioni. On feedback stabilisation of switched linear systems via Lie-algebraic techniques. In 48th IEEE Conf. on Decision and Control, Shanghai, China, 2009.

[7] H. Haimovich, E. Kofman, and M. M. Seron. Systematic ultimate bound computation for sampled-data systems with quantization. $\mathrm{Au}$ tomatica, 43(6):1117-1123, 2007.

[8] H. Haimovich, E. Kofman, and M.M. Seron. Analysis and improvements of a systematic componentwise ultimate-bound computation method. In 17th IFAC World Congress, Seoul, South Korea, 2008.

[9] R.A. Horn and C.R. Johnson. Topics in matrix analysis. Cambridge University Press, 1991.

[10] H. Khalil. Nonlinear Systems. Prentice-Hall, New Jersey, 2002. 3rd.ed.

[11] E. Kofman, F. Fontenla, and H. Haimovich. Cómputo por componentes del error introducido por señales PWM en sistemas LTI. In Congr. Argentino de Control Automático (AADECA), Buenos Aires, Argentina, 2008.

[12] E. Kofman, H. Haimovich, and M. M. Seron. A systematic method to obtain ultimate bounds for perturbed systems. International Journal of Control, 80(2):167-178, 2007.

[13] E. Kofman, M. M. Seron, and H. Haimovich. Control design with guaranteed ultimate bound for perturbed systems. Automatica, 44(7):1815-1821, 2008. doi:10.1016/j.automatica.2007.10.022.

[14] D. Liberzon. Switching in systems and control. Boston, MA: Birkhauser, 2003.

[15] D. Liberzon and S. Morse. Basic problems in stability and design of switched systems. Control Systems Magazine, October 1999.

[16] H. Lin and P.J. Antsaklis. Stability and persistent disturbance attenuation properties for a class of networked control systems: switched system approach. International Journal of Control, 78(18):1447-1458, 2005.

[17] H. Lin and P.J. Antsaklis. Stability and stabilizability of switched linear systems: a survey of recent results. IEEE Trans. on Automatic Control, 54(2):308-322, 2009.

[18] S. Olaru, J.A. De Doná, and M.M. Seron. Positive invariant sets for fault tolerant multisensor control schemes. In Proceedings 17 IFAC World Congress, Seoul, Korea, July 2008.

[19] A. Sala. Computer control under time-varying sampling period: an LMI gridding approach. Automatica, 41(12):2077-2082, 2005.

[20] M.M. Seron, J.A. De Doná, and J.J. Martínez. Invariant set approach to actuator fault tolerant control. In 7th IFAC Symposium on Fault Detection, Supervision and Safety of Technical Processes, SAFEPROCESS'09, Barcelona, Spain, 30 June to 3 July 2009.

[21] M.M. Seron, X.W. Zhuo, J.A. De Doná, and J.J. Martínez. Multisensor switching control strategy with fault tolerance guarantees. Automatica, 44(1):88-97, 2008.

[22] R. Shorten, F. Wirth, O. Mason, K. Wulff, and C. King. Stability criteria for switched and hybrid systems. SIAM Review, 49(4):545592, 2007.

[23] R. Su, S. Abdelwahed, and S. Neema. Computing finitely reachable containable region for switching systems. IEE Proc.-Control Theory Appl., 152(4):477-486, 2005.

[24] J. Theys. Joint spectral radius: theory and approximations. $\mathrm{PhD}$ thesis, Center for Systems Engineering and Applied Mechanics, Université catholique de Louvain, 2005.

[25] X. Xu, G. Zhai, and S. He. On practical asymptotic stabilizability of switched affine systems. Nonlinear Analysis: Hybrid Systems, 2:196208, 2008.

[26] A. Yetendje, M.M. Seron, and J.A. De Doná. Fault-tolerant switching control of a magnetic levitation system. In 7th IFAC Symposium on Fault Detection, Supervision and Safety of Technical Processes, SAFEPROCESS'09, Barcelona, June 30 to July 3, 2009.

[27] L. Zhang, Y. Chen, Z. Sun, N.E. Mastorakis, and A.Y. Alexsandrov. Uniformly ultimate boundedness control for switched linear systems with parameter uncertainties. In Proc. American Control Conference, pages 3971-3974, Seattle, Washington, USA, 2008.

[28] L. Zhang, Y. Lu, Y. Chen, and N.E. Mastorakis. Robust uniformly ultimate boundedness control for uncertain switched linear systems. Computers and Mathematics with Applications, 56:1709-1714, 2008.

[29] X. Zhang and J. Zhao. An algorithm of ultimate boundedness for a class of switched linear systems. International Journal of Control, 75(16/17):1399-1405, 2002. 